\title{
Determinação da produção de metalo- $\beta$-lactamases em amostras de Pseudomonas aeruginosa isoladas em João Pessoa, Paraíba
}

\author{
Detection of metallo-ß-lactamase producing Pseudomonas aeruginosa isolated in João Pessoa, Paraíba, Brazil
}

\author{
Lauro Santos Filho ${ }^{1}$ \\ Isabele Beserra Santos ${ }^{2}$ \\ Alexandro Mangueira Lima de Assis ${ }^{2}$ \\ Danilo Elias Xavier ${ }^{2}$
}

\begin{abstract}
unitermos resumo
Pseudomonas aeruginosa

Bactérias produtoras de metalo- $\beta$-lactamases (MBLs) são em grande parte resistentes aos

Resistência

betalactâmicos de largo espectro, incluindo oximino-aminotiazol cefalosporinas e também

Metalo- $\beta$-lactamases aos carbapenens. O objetivo deste trabalho foi detectar cepas de Pseudomonas aeruginosa resistentes ao imipenem e à ceftazidima, assim como identificar aquelas produtoras de MBLs. Foram estudadas 198 linhagens não-repetitivas isoladas de diversas amostras clínicas, hospitalares e comunitárias, identificadas bioquimicamente por técnicas de rotina. A triagem para a detecção de amostras produtoras de MBLs foi realizada pelo método de dupla difusão proposto por Arakawa et al. (2000) e modificado por Nakajima et al. (2001), utilizando discos contendo mercaptoacetato de sódio. Foi detectado um percentual de resistência de 19,7\% (39/198) ao imipenem e de 15,2\% (30/198) à ceftazidima, com 10,1\%(20/198)

de resistência cruzada aos dois antimicrobianos. Entre estas amostras, 2\% (4/198)

demonstraram produção de MBLs e padrão de multirresistência. A detecção destas amostras

configura um problema emergente, com importantes implicações na terapêutica

antimicrobiana, necessitando, portanto, de maior investigação através de metodologia

molecular, para melhor caracterizar a extensão do problema.
\end{abstract}

\section{abstract}

Producers of metallo- $\beta$-lactamases (MBLs) have been shown to confer resistance to carbapenems.

The present study was undertaken to evaluate the presence of resistance to imipenem and oxymino cephalosporins, as well as MBLs production among clinical isolates of Pseudomonas

aeruginosa in João Pessoa-PB, Brazil. A total of 198 non-repetitive strains of $\mathrm{P}$. aeruginosa identified by routine techniques were studied. These strains were screened for MBL production, using the double disk diffusion test previously described by Arakawa et al. (2000), as modified by

Nakajima et al. (2001), using disks containing sodium mercaptoacetate as inhibitor of MBL. Strains were considered to be MBL producer when they demonstrated a typical expansion in the inhibitory zone by the presence of thiol compound. A total of four strains (2\%) showed positive results among the isolates tested. Our findings indicate that the appearance of metallo- $\beta$ lactamase in Pseudomonas aeruginosa strains is an emerging problem, with important therapeutic implications, and this fact needs further investigation including the use of molecular methodologies to fully characterize the extent of the problem.

\section{key words}

Pseudomonas aeruginosa

Resistance

Metallo-B-lactamases 


\section{Introdução}

Bactérias gram-negativas resistentes ao carbapenem, incluindo linhagens de enterobactérias e de Pseudomonas aeruginosa, têm sido isoladas freqüentemente, com capacidade de produzir metalo- $\beta$-lactamases (MBLs), que são enzimas com atividade sobre vários betalactâmicos, incluindo cefamicinas e carbapenens, e ainda sobre os inibidores de betalactâmicos, como ácido clavulânico e sulbactam $(1,4)$.

A propagação e a disseminação desse tipo de microrganismo têm sido confirmadas em vários locais, tais como Japão, Itália, Cingapura, Inglaterra, Portugal, Grécia e Taiwan $(5,6,8,16,17,20)$. Em nosso meio, foram isoladas as primeiras amostras no Hospital Universitário da Universidade Federal do Rio de Janeiro, não havendo, até o momento, registro de isolamento em outras regiões (11).

As MBLs pertencem ao grupo 3 de $\beta$-lactamases de espectro ampliado, fazendo parte de uma classe funcional comum de metaloenzimas classificadas com base em sua habilidade de hidrolisar o imipenem em um nível mensurável e em sua característica de serem inibidas por íns de metal quelante, como o EDTA, assim como em sua suscetibilidade aos inibidores de betalactamases disponíveis comercialmente $(4,12)$.

Um crescente número de enzimas que hidrolisam carbapenens foi descrito nos últimos anos, especialmente no Japão, onde os carbapenêmicos são líderes de mercado entre os antimicrobianos $\beta$-lactâmicos parenterais, em contraste com outras áreas geográficas, onde os carbapenens são usados em bases mais restritas $(12,19)$. Talvez este fato explique o maior isolamento dessas amostras em países asiáticos (13).

Carbapenens são antimicrobianos usados geralmente como drogas de reserva no tratamento de infecções causadas por bactérias gram-negativas resistentes a outros agentes $\beta$-lactâmicos, considerando seu espectro de atividade e estabilidade à hidrólise pela maioria das $\beta$-lactamases, incluindo as betalactamases de espectro ampliado (ESBL) (17). Desse modo, é recomendada a restrição no uso de cefamicinas e de carbapenens como medida de prevenção da disseminação de bactérias gramnegativas do tipo Serratia marcescens ou Pseudomonas aeruginosa com capacidade de produzir metalo$\beta$-lactamase (13).

Mais recentemente foram descritas, em Hong Kong, Cingapura, Taiwan e Japão, amostras de Acinetobacter baumanii $(15,18)$ e Klebsiella pneumoniae $(7,8)$ apresentando resistência múltipla aos antimicrobianos e, também, com capacidade de produzir metalo- $\beta$-lactamase.

Como os produtores de MBLs tendem a demonstrar resistência às drogas $\beta$-lactâmicas de largo espectro, incluindo oximino cefalosporinas, cefamicinas e carbapenens, sua detecção preliminar é importante para o controle de infecções e a orientação adequada da terapêutica (3). Com esta finalidade, a análise por reação de polimerase em cadeia (PCR) é a técnica mais adequada e satisfatória para a confirmação de amostras MBL-positivas. No entanto este método tem seu uso prático limitado em virtude do elevado custo (13), sendo necessário, portanto, um método simples, específico e de baixo custo que possa ser utilizado em rotina de laboratório (9).

Arakawa et al. (2000) testaram vários produtos químicos inibidores das metalo- $\beta$-lactamases, estabelecendo um método conveniente de difusão com duplo disco para triagem de bactérias produtoras de MBL, através do uso do ácido 2-mercapto propiônico (2-MPA). Entre os inibidores de MBL usados, o 2-MPA apresentou os melhores resultados, pelo fato de este agente químico bloquear a atividade mais efetivamente, mesmo em baixa concentração. No entanto seu uso é limitado, em virtude de sua alta volatilidade (14).

O antimicrobiano ceftazidima (CTZ) pareceu ser o substrato mais adequado para este teste, considerando-se que amostras produtoras de MBL (IMP-1) usualmente demonstram alto nível de resistência à CTZ (MIC $>64 \mathrm{mg} / \mathrm{ml})$, fato confirmado em estudos anteriores em que um considerável efeito inibitório dos compostos tiólicos foi geralmente observado (13). O método de difusão usando-se o ácido etilenodiaminotetracético (EDTA) para triagem dessas amostras tem sido reportado. Contudo o EDTA inibe, por si mesmo, o crescimento bacteriano de algumas espécies ou linhagens bacterianas, dificultando a interpretação dos resultados obtidos (1).

Considerando os fatos descritos, e com finalidade de contornar alguns problemas técnicos, foi proposto um teste usando-se o mercaptoacetato de sódio (SMA - Eiken Chemical, Japão), um composto estabilizado que pode ser incorporado a discos de papel-filtro, na concentração de $3 \mathrm{mg} /$ disco, sendo conveniente para triagem de MBLs em rotina de laboratórios clínicos (9). Além disso, o mercaptoacetato de sódio apresenta um forte efeito inibitório nas MBLs, associado a uma baixa atividade bactericida (MIC $>400 \mathrm{mg} / \mathrm{ml}$ ), conferindo ao teste maior especificidade. 


\section{Objetivos}

O objetivo fundamental do presente estudo foi determinar a presença de linhagens de Pseudomonas aeruginosa produtoras de metalo- $\beta$-lactamases.

Como objetivos secundários temos:

- triagem preliminar fenotípica de amostras resistentes ao imipenem e à ceftazidima;

- avaliação da utilização de teste de dupla difusão usando discos contendo ceftazidima (CTZ) e mercaptoacetato de sódio (SMA);

- orientação para o tratamento com antimicrobianos contra este tipo de linhagem bacteriana.

\section{Metodologia}

\section{Amostras bacterianas}

Foi utilizado um total de 198 amostras não-repetitivas de Pseudomonas aeruginosa de origem comunitária ou hospitalar, provenientes de diversos sítios anatômicos, isoladas em laboratórios clínicos de João Pessoa (PB), no período de janeiro a novembro de 2000.

\section{Processamento de laboratório}

O processamento laboratorial das amostras foi realizado no Laboratório de Microbiologia do Núcleo de Medicina Tropical/CCS/Universidade Federal da Paraíba, e as amostras de Pseudomonas aeruginosa foram identificadas por métodos de rotina.

\section{Triagem preliminar das amostras}

- O teste de sensibilidade aos antimicrobianos foi realizado utilizando-se as seguintes drogas: amicacina (AMI), aztreonam (ATM), cefepima (CPM), ceftazidima (CTZ), cefoperazona (CFP), ciprofloxacino (CIP), colistina (CT), gentamicina (GEN), imipenem (IMI), piperacilina (PIP) e ticarcilina/ácido clavulânico (TIM) (Oxoid);

- foram consideradas para estudo as amostras que mostraram perfil de resistência elevado, com diminuição de sensibilidade ao imipenem (diâmetro de inibição $<16 \mathrm{~mm}$ ) e à ceftazidima (diâmetro de inibição $<18 \mathrm{~mm}$ ), verificado no antibiograma de rotina realizado pelo método de difusão com discos (10). Foram selecionadas, para testes de detecção de MBLs, as amostras que apresentaram resistência cruzada aos dois antibióticos.

\section{Determinação presuntiva da produção de MBLs}

- Amostras: uma colônia de cada linhagem bacteriana selecionada foi diluída em caldo Mueller-Hinton (MH Broth, Oxoid) para se obterem $10^{6} \mathrm{UFC} / \mathrm{ml}$, sendo então espalhada com auxílio de swab em uma placa de Petri $(10 \mathrm{~mm} \times 100 \mathrm{~mm})$, de acordo com o protocolo recomendado pelo National Committee for Clinical Laboratory Standards (NCCLS) (1999);

- dois discos comerciais de CTZ (Oxoid), cada um contendo $30 \mathrm{mg}$, foram então colocados sobre as placas inoculadas. A distância entre os dois discos foi de aproximadamente $30 \mathrm{~mm}$ (Figura 1). Um disco padronizado, contendo $3 \mathrm{mg}$ de mercaptoacetato de sódio (SMA Eiken Chemical, Japão), foi colocado $15 \mathrm{~mm}$ abaixo do segundo disco de CTZ (9).

A amostra teste foi considerada produtora de MBL quando uma diferença de $5 \mathrm{~mm}$ ou mais foi observada entre os diâmetros da zona de inibição, comparando-se os dois discos de CTZ. Não foram incluídas cepas padrão de controle.

\section{Resultados e discussão}

Foi detectado um percentual de resistência de 19,7\% (39/ 198) ao imipenem e de $15,2 \%$ (30/198) à ceftazidima, com 10,1\% (20/198) de amostras apresentando resistência cruzada aos dois antimicrobianos. Entre estas amostras, 2\% (4/198) demonstraram produção de metalo- $\beta$-lactamases através do método presuntivo de disco aproximação descrito por Arakawa et al. (2000) e modificado por Nakajima et al. (2001), que apresenta uma correlação de $94 \%$ com a análise molecular usando-se a técnica de PCR (13).

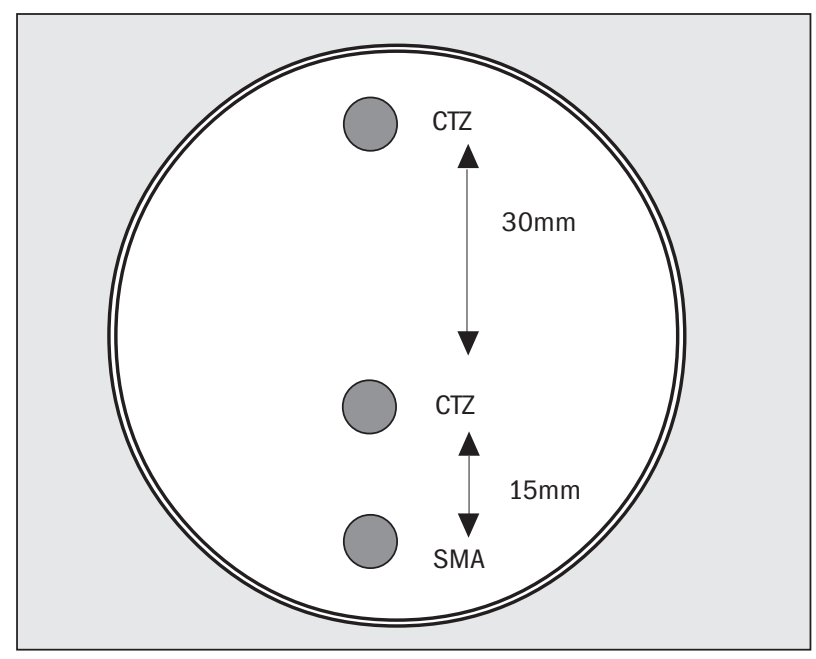

Figura 1 - Diagrama de colocação dos discos para o teste de disco aproximação usando-se SMA 
Estabelecendo-se uma comparação entre as amostras de origem comunitária e as de origem hospitalar, observam-se, na Figura 5, as percentagens de amostras de acordo com o local de origem. No total, tem-se 52\% de amostras de origem hospitalar e $48 \%$ de origem comunitária. No entanto, quando se consideram apenas as amostras multirresistentes, este percentual de amostras hospitalares se eleva para $85 \%$.

A maior parte das amostras $(39,4 \%)$ foi proveniente do trato respiratório, seguido do trato urinário $(24,2 \%)$ e de escaras (16,3\%) (Figura 6).

\section{Compostos Thiol (mercapto-)}

- Ácido 2-mercaptopropiônico $\mathrm{PM}=106,14$

$\mathrm{CH}_{3}-\mathrm{CH}-\mathrm{COOH}$

$\mathrm{SH}$

- Mercaptoacetato de sódio $\quad P M=114,10$

$\mathrm{SH}-\mathrm{CH}_{2}-\mathrm{COONa}$

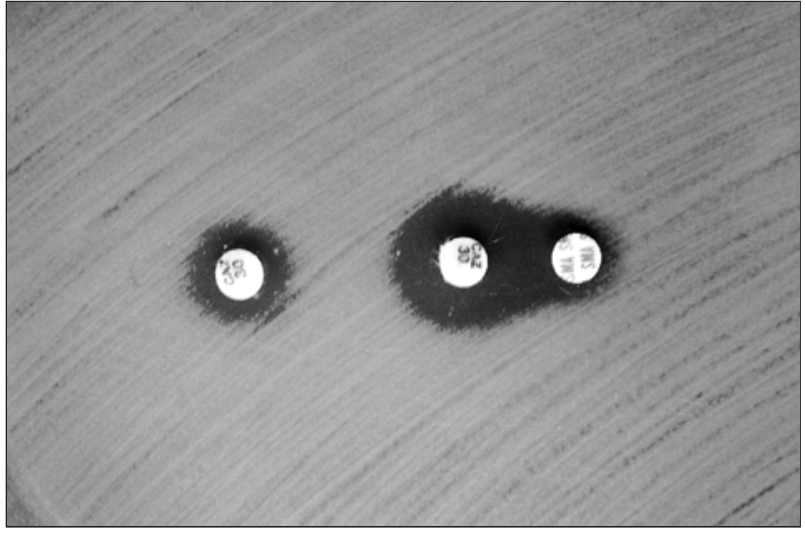

Figura 3 - Teste positivo: identificação presuntiva de produção de metalo$\beta$-lactamase

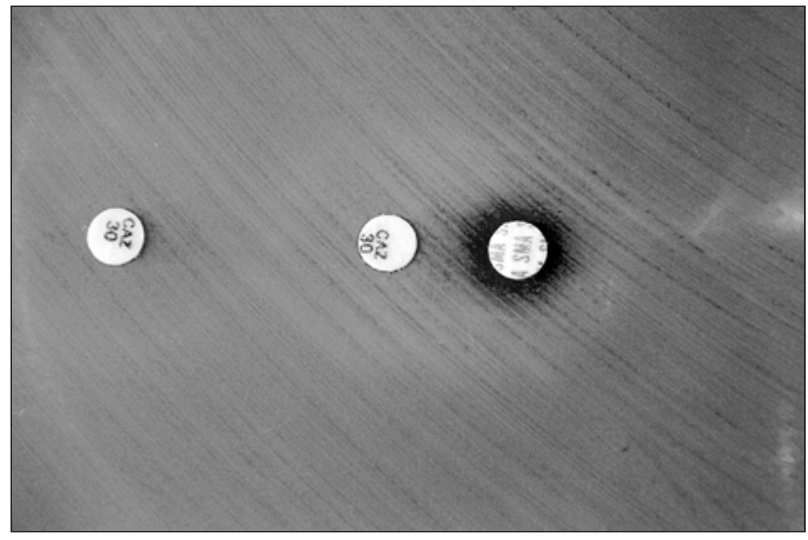

Figura 4 - Teste negativo: crescimento bacteriano permanece inalterado

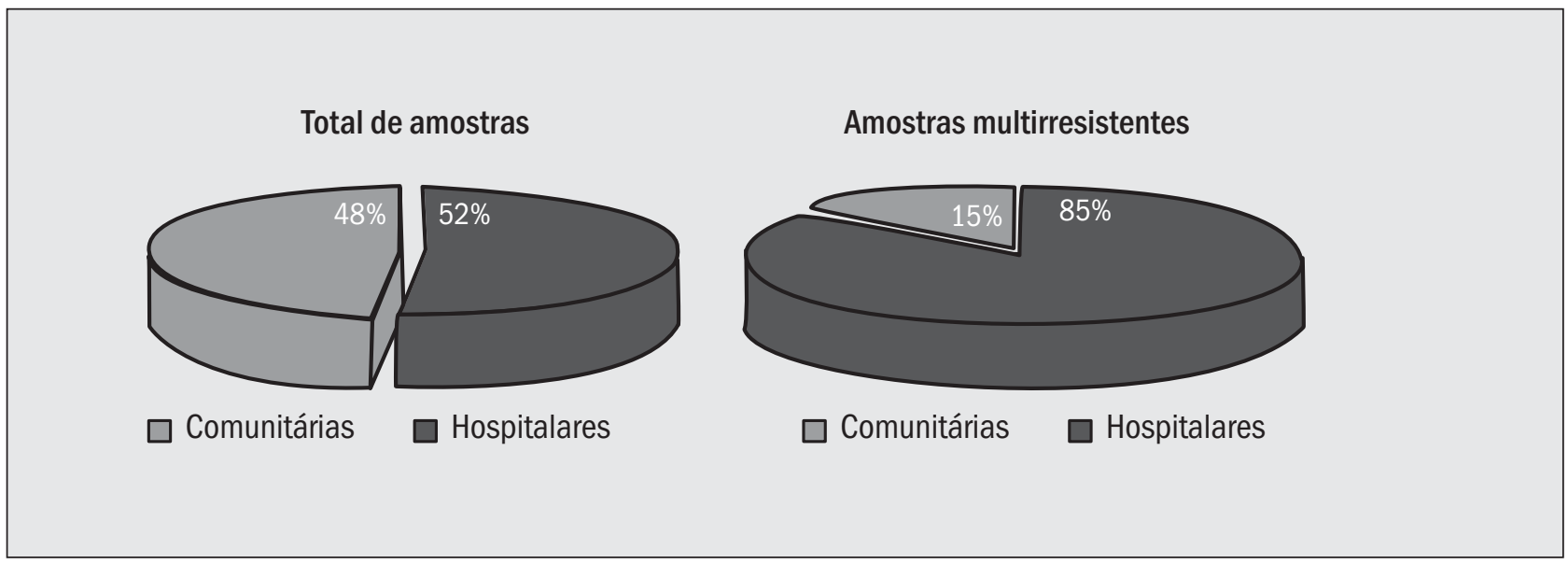

Figura 5 - Origem das 198 amostras de P. aeruginosa testadas 


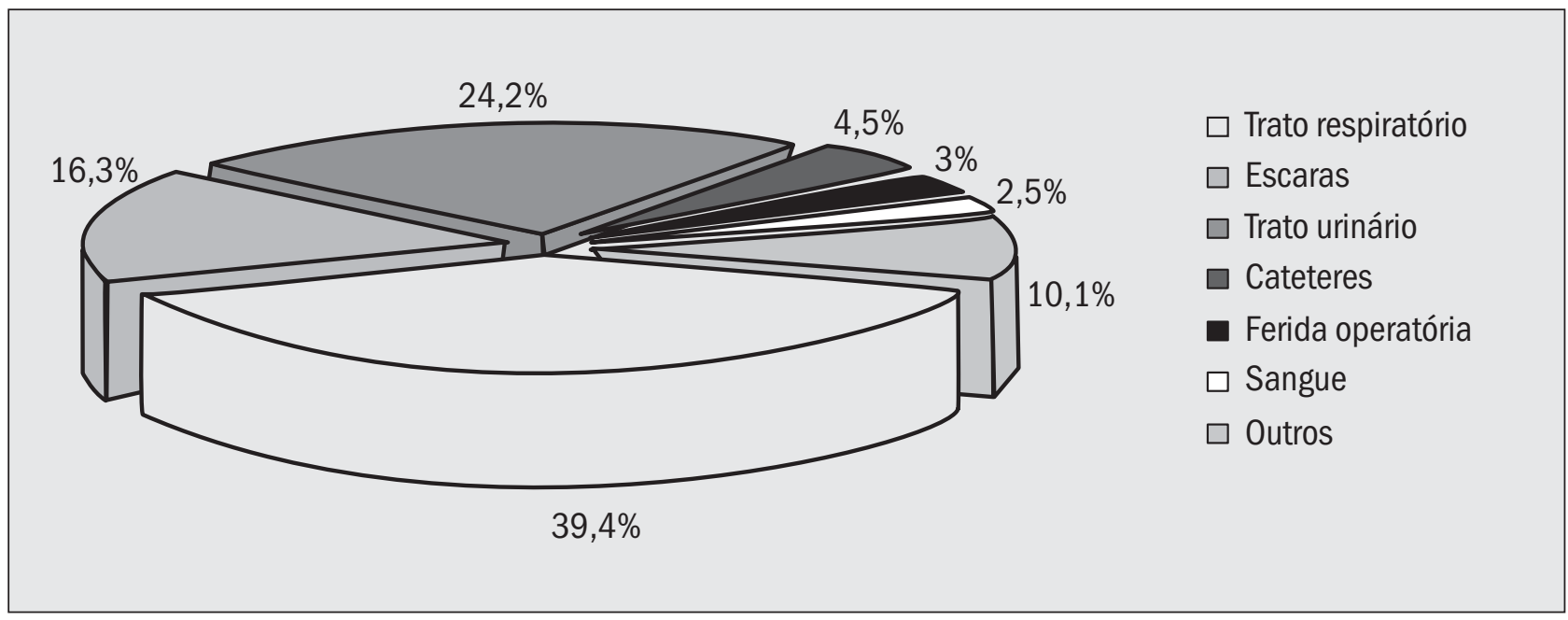

Figura 6 - Locais de isolamento das 198 amostras de P. aeruginosa testadas

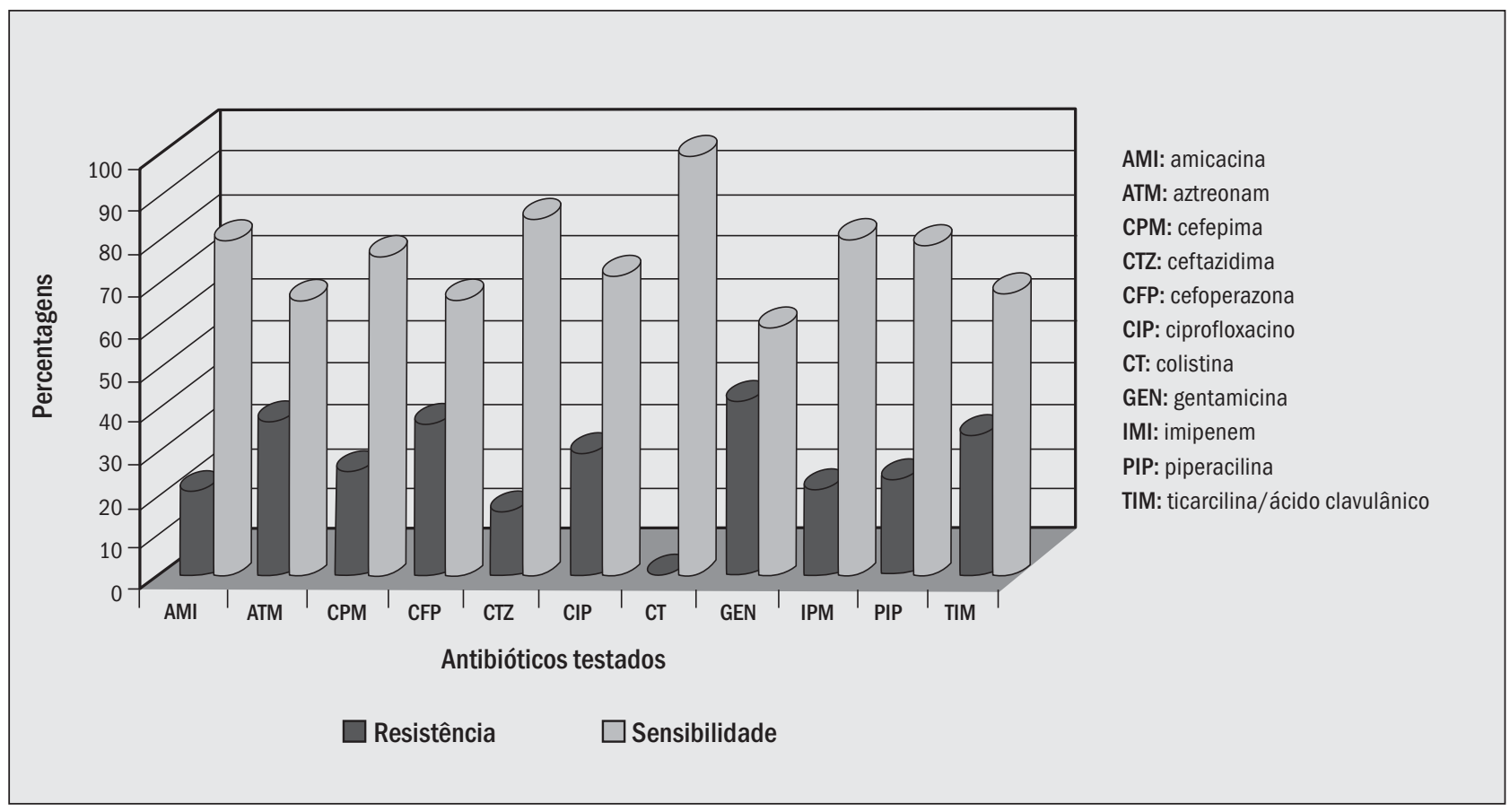

Figura 7 - Padrão de resistência de 198 cepas de Pseudomonas aeruginosa isoladas em laboratórios clínicos de João Pessoa (PB)

\section{Conclusões}

Com o relato do aparecimento recente de infecções causadas por bactérias produtoras de MBLs na Ásia e na Europa, o registro destas amostras no Brasil, mais precisamente no Nordeste, merece uma atenção especial no tocante à possibilidade de surgimento de novos casos em um futuro próximo, alterando a conduta terapêutica nestas infecções e servindo, ainda, de alerta para motivar ações necessárias à prevenção da disseminação destes microrganismos.
Configura-se a necessidade da utilização, em rotina de laboratórios clínicos, de testes para a detecção preliminar de linhagens produtoras de MBLs que sejam práticos e aplicáveis, como o método proposto neste estudo.

A confirmação dos casos e um estudo epidemiológico devem ser conduzidos utilizando-se, com esta finalidade, técnicas moleculares de análise.

Este método, usando-se o disco contendo mercaptoacetato de sódio (SMA), apresenta a característica de ter alta reprodutibilidade, facilidade de execução e baixo custo, tendo ainda a vantagem de ser um composto estável, 
com validade prolongada à temperatura ambiente e com baixa ação bactericida.

\section{Agradecimentos}

Ao dr. Kazuhiro Nakajima (Microbiological Reagents Dept., Eiken Chemical Co., Japão), que gentilmente nos cedeu os discos padronizados de mercaptoacetato de sódio.

Aos colegas microbiologistas dos laboratórios clínicos de João Pessoa que nos forneceram amostras bacterianas utilizadas neste estudo: dr. Mario Cadena Bieda, dra. Bernadete Helena Cavalcanti dos Santos e dra. Maria Marluce Vasconcelos Castro.

\section{Referências}

I. Arakawa, Y. et al. Convenient test for screening metallo- $\beta$ lactamase: producing gram-negative bacteria by using thiol compounds. J. Clin. Microbiol., 38: 40-3, 2000.

2. Arakawa, Y. et al. Detection of VIM-2 metallo- $\beta$-lactamase producing Pseudomonas by thiol compounds in Japan. Abstract A-101. In: 10 ${ }^{\text {th }}$ ASM General Meeting, Orlando, 2001 .

3. Bush, K. Metallo-betalactamases: a class apart. Clin. Infect. Dis., 27: S48-S53, 1998.

4. Bush, K.; Jacoby, G.A. \& Medeiros, A.A. A functional classification scheme for $\beta$-lactamases and its correlation with molecular structure. Antimicrob. Agents Chemother., 39: 121 1-33, 1995.

5. Cardoso, O. et al. Carbapenem-hidrolozing from clinical isolates of Pseudomonas aeruginosa in Portugal. J. Antimicrob. Chemother, 44: 135-8, 1999.

6. Cornaglia, G. et al. Appearance of IMP-I metallo- $\beta$-lactamase in Europe. Lancet, 353: 899-900, 1999.

7. Jing-Jou, Y.; Wen-Chien, K. \& Jiunn-Jong, W. Identification of a plamid encoding SHV-I2, TEM-I, and a variant of IMP-2 metallo- $\beta$-lactamase, IMP-8, from a clinical isolate of Klebsiella pneumoniae. Antimicrob. Agents Chemother, 45: 2368-7I, 200।.

8. Koh, T.H. et al. Carbapenem-hydrolysing IMP-I $\beta$-lactamase in Klebsiella pneumoniae in Cingapore. Lancet, 353: 2162-4, 1999.

9. Nakajima, K. et al. Disk diffusion and microdilution tests for screening metallo- $\beta$-lactamase producing bacteria. Abstract

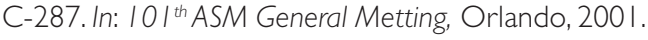

10. National Committee for Clinical Laboratory Standards. Methods for dilution antimicrobial susceptibility tests for bacteria that grow aerobically. Approved standard M7-A4. National Committee for Clinical Laboratory Standards, Wayne, PA, 1999.
I।. Pellegrino, F.L.P. et al. Antimicrobial resistance and genotype characterization of Pseudomonas aeruginosa isolates from a university affiliated hospital in Rio de Janeiro. Abstract L14. In: 10 I $^{\text {th }}$ ASM General Metting, Orlando, 200 I.

12. Rasmussen, B.A. \& Bush, K. Carbapenem-hidrolizing- $\beta$ lactamases. Antimicrob. Agents Chemother, 41:223-31, 1997.

13. Senda, K. et al. PCR detection of metallo- $\beta$-lactamase gene (bla|MP) in gram-negative rods resistant to broad-spectrum B-lactams. J. Clin. Microbiol., 34: 2909- I3, 1996.

14. Shibata, N. et al. Molecular epidemiology of metallo- $\beta$-lactamase among gram-negative rods detected by thiol compounds in Japan. Abstract C-524. In: $10^{\text {th }}$ ASM General Metting, Orlando, 2001.

15. Takahashi, A. et al. Detection of carbapenase-producing Acinetobacter baumanii in a hospital. J. Clin. Microbiol., 38: 526-9, 2000.

16.Tsakis, A. et al. Outbreak of infections caused by Pseudomonas aeruginosa producing VIM-I carbapenemase in Greece. J. Clin. Microbiol., 38: I290-2, 2000.

17.Yan, J.J. et al. Emergence of a novel variant of VIM-2 metallo- $\beta$ lactamase (VIM-3) in Pseudomonas aeruginosa in Taiwan. Abstract A- 105. In: I 0 th ASM General Meeting, Orlando, 2001.

18. Yiu-Wai, C. et al. IMP-4, a novel metallo- $\beta$-lactamase from nosocomial Acinetobacter spp. collected in Hong Kong between 1994 and 1998. Antimicrob. Agents Chemother, 45: 7|0-4, 200।.

19. Watanabe, M. et al. Transferable imipenem resistance in Pseudomonas aeruginosa. Antimicrob. Agents Chemother, 35: |47-5 |, 199|.

20. Woodford, N. et al. Carbapenemase-producing Pseudomonas aeruginosa in UK. Lancet, 352: 546-7, 1998. 